\title{
Migração, Seleção e Diferenciais de Rendimentos do Trabalho no Centro-Oeste do Brasil - 2000/2010
}

Migration, Selection and Labor Income Differential in Brazil's Center-West - 2000/2010

\author{
Luís Abel da Silva Filho \\ Patrick Leite Santos ${ }^{\mathrm{b}}$ \\ Denis Fernandes Alves ${ }^{c}$ \\ Bruno César Brito Miyamoto ${ }^{d}$ \\ Jaim José da Silva Junior ${ }^{\mathrm{e}}$
}

\begin{abstract}
Resumo: Este artigo tem como objetivo testar a hipótese de seleção positiva migratória intermunicipal na região Centro-Oeste do Brasil. Como abordagem empírica é estimado um modelo econométrico a partir do método de seleção amostral de Heckman utilizando-se os dados de migração intermunicipal extraídos dos Censos Demográficos do Brasil nos anos 2000 e 2010. Os resultados mostram que o migrante intermunicipal da região Centro-Oeste do Brasil é positivamente selecionado e que as características não observáveis afetam a decisão de migração.
\end{abstract}

Palavras-chave: Migração; Seleção; Centro-Oeste.

Classificação JEL: J0. J61.

\begin{abstract}
This article aims to test the hypothesis of positive intermunicipal migration selection in the Central-West region of Brazil. As an empirical approach, an econometric model is estimated from Heckman's sample selection method using the intermunicipal migration data obtained by the Demographic Census of Brazil in 2000 and 2010. The results show that the intermunicipal migrant from the Central-West region of Brazil is positively selected and that the unobservable productive characteristics affect migration decision.
\end{abstract}

Keywords: Migration; Selection; Center-West.

JEL Classification: J0. J61.

\footnotetext{
${ }^{\text {a }}$ Professor Adjunto do Departamento de Economia da Universidade Regional do Cariri - URCA. E-mail: abeleconomia@hotmail.com

${ }^{\mathrm{b}}$ Doutorando em Economia pelo PPGE/UFU. E-mail: patrickeconomia@ hotmail.com

${ }^{\mathrm{c}}$ Doutorando em Economia pelo PIMES/UFPE. E-mail: denis_fernandes@outlook.com

${ }^{\mathrm{d}}$ Professor do Instituto Federal do Rio Grande do Sul. E-mail: miyamototup@ gmail.com

${ }^{\text {e }}$ Professor Adjunto da Faculdade de Agronomia e Medicina Veterinária da Universidade de Brasília- UnB. Email: jaim@unb.br
} 


\section{Introdução}

Transformações estruturais na economia brasileira, ao longo dos anos, vêm sendo responsáveis pelas configurações na produção e no trabalho ao largo do território nacional. Os últimos Censos Demográficos do Brasil - 1991, 2000 e 2010 - mostram configurações relativamente novas na dinâmica migratória interna (IBGE, 2010). As regiões economicamente dinâmicas e tradicionalmente receptoras de migrantes de todas as partes do país vêm perdendo participação, relativamente, para outras regiões que se despontam na atração de migrantes ao longo dos anos.

No Brasil, a região Centro-Oeste se destaca como destino da mão de obra de outras regiões, inicialmente entre os anos de 1956 e 1960, com a construção de Brasília, e posteriormente, nos últimos Censos (2000 e 2010), para suprir as necessidades de mão de obra para a manutenção e funcionamento das instituições públicas que se concentraram na capital do Brasil, assim como nas atividades e serviços que se desenvolveram na região, a partir dessa nova demanda, com encadeamentos para frente e para trás. Além do setor público, a região tem atraído trabalhadores para o agronegócio, que tem se desenvolvido e se estabelecido como um dos mais competitivos do Brasil, tanto em grãos quanto em pecuária, com grande intensidade de capital e tecnologia.

Conforme dados das contas regionais, disponibilizado pelo IBGE, em termos de participação no PIB nacional, o Centro-Oeste aumentou sua parcela de 7,2\%, em 2000, para $9,1 \%$ do PIB nacional, em 2010. No mesmo período, a população residente da região cresceu acima da média nacional, saindo de 11.638 .658 para 14.058.094 milhões de habitantes, total equivalente a uma taxa de crescimento de $20 \%$. Já a população ocupada na região cresceu aproximadamente $44 \%$ no período entre os Censos Demográficos de 2000 e 2010. A escolha da região e do período deveu-se, ainda, em função do seu processo recente de desenvolvimento socioeconômico, fortemente marcado por processos migratórios, ligados tanto à expansão da fronteira agrícola como à ocupação urbana.

Assim sendo, este artigo visa estudar a dinâmica migratória e testar a hipótese de seleção migratória positiva na região Centro-Oeste do Brasil, entre os anos de 2000 e 2010. Para isso, foi estimado um modelo econométrico a partir do método com correção de viés de seleção amostral proposto por Heckman (1979), utilizando-se os dados cross-section de migração intermunicipal auferidos pelos Censos Demográficos do Brasil nos anos $2000 \mathrm{e}$ 2010, disponibilizados pelo Instituto Brasileiro de Geografia e Estatística (IBGE). O modelo busca capturar a probabilidade com que a mão de obra qualificada e dotada de demais características positivas contribui para o aumento do capital humano e desenvolvimento econômico da região.

Para atingir o objetivo proposto, o artigo está estruturado em cinco seções. Além destas considerações iniciais. A segunda seção aborda o referencial teórico que serviu de base para o estudo. A terceira seção apresenta os procedimentos metodológicos desenvolvidos no artigo. Na quarta seção divulgam-se e analisam-se os principais resultados obtidos, sendo que na primeira parte faz-se uma discussão com base em estatísticas descritivas da população ocupada nesta região e a segunda parte da seção 
apresenta os resultados do modelo que estima a probabilidade de migração e os diferenciais de rendimentos dos migrantes, segundo as características socioeconômicas e demográficas. Por fim, na quinta seção traçam-se as considerações finais e as perspectivas de novas abordagens.

\section{Determinantes da Dinâmica Migratória: uma revisão da literatura}

A literatura econômica internacional e nacional que estuda a migração, seleção e diferenciais de rendimentos tem como pano de fundo entender quais são os fatores determinantes da decisão de migrar e quais são os impactos desses na dinâmica migratória. Nesse contexto, duas teorias têm predominância no debate, dada a potencialidade dos argumentos: a teoria do capital humano e a teoria da segmentação ou dualidade do mercado de trabalho. Entre essas, a ótica da decisão individual é a mais difundida e concentra o maior número de pesquisas empíricas realizadas (SANTOS et al., 2010; GOLD \& NAWYN, 2019).

$\mathrm{Na}$ literatura econômica internacional, a discussão predominante, que inseriu a questão da migração na ótica da teoria do capital humano, compreende a migração como um investimento feito por agentes que buscam, em seus lugares de destino, remuneração superior àquela auferida no lugar de origem (MASSEY, 1993; WHITE, 2016). Nesta perspectiva, a migração é, além de uma forma de mobilidade da força de trabalho, um investimento em capital humano, que resulta do conhecimento e experiência adquiridos pelos migrantes durante a transição e nos locais de destino (JOHNSON \& SALT, 2018).

Por este aspecto, entende-se que a migração produz resultados seletivos sobre o capital humano em âmbito regional. Uma vez que os indivíduos migrantes detêm características não observáveis que afetam a decisão de migrar, adota-se o pressuposto de que a força de trabalho migrante é mais ambiciosa, tomadora de risco, determinada e persistente que indivíduos não migrantes com características socioeconômicas observáveis semelhantes (BORJAS, 1987; DUSTMANN \& GLITZ, 2011). Neste contexto, os custos subjetivos da migração são superados e há uma seleção positiva dos migrantes que contribui para a elevação do capital humano e do desenvolvimento regional (LEE, 1966; RAVENSTEIN, 1980; BASTIA \& SKELDON, 2019).

A teoria do capital humano é a pioneira do debate, tendo como núcleo de análise o poder de determinação de migrar atribuído às características pessoais dos indivíduos. $\mathrm{Na}$ literatura internacional, a investigação dos determinantes a partir dessa ótica identificou que a migração é tratada como um investimento para obtenção de rendimentos superiores, à medida que o indivíduo migrante acumula conhecimento e experiência resultantes do ato de migrar (MASSEY, 1993; WHITE, 2016; JOHNSON \& SALT, 2018). Nesse sentido, os migrantes possuem características não observáveis, como ambição e maior disposição a risco, que desencadeiam em resultados seletivos positivos da decisão de migrar e que podem favorecer o desenvolvimento da região de destino (LEE, 1966; RAVENSTEIN, 1980; BORJAS, 1987; DUSTMANN \& GLITZ, 2011; BASTIA \& SKELDON, 2019). 
Em relação às características socioeconômicas observáveis, Mincer (1978) ressalta que as características familiares (tamanho da família, características do chefe do domicílio, entre outras) dos indivíduos são importantes determinantes da migração. Ele explica que os indivíduos que possuem familiares dependentes, e, portanto, maior nível de responsabilidade dentro da família - como indivíduos casados ou na posição de chefe -, ou que possuem alto nível de dependência - como filhos -, possuem menor probabilidade de migrar. Becker (1993) coloca o nível de instrução formal do indivíduo como um dos principais determinantes da probabilidade de migrar e do sucesso dessa migração - o segundo em termos de renda. Segundo o autor, quanto maior o nível de instrução, maior tende a ser a probabilidade de migração e maior tende a ser o retorno monetário.

Assim sendo, as decisões de migração são atribuídas às questões de naturezas individuais que se dão no núcleo da família e que afetam diretamente a decisão de buscar oportunidade de inserção ocupacional em outras áreas geoeconômicas. Envolto a estes atributos de natureza individual estão acopladas importantes características socioeconômicas observáveis dos indivíduos, tais como: nível de instrução formal, tamanho dos arranjos familiares, número de filhos menores de idade; além das características não observáveis como ambição, persistência, entusiasmo, entre outras (SILVA FILHO, 2017). Nessa perspectiva teórica, a decisão de migração é uma decisão de natureza individual e não está diretamente ligada a questões de natureza estrutural do mercado de trabalho.

Já a teoria da segmentação ou dualidade do mercado de trabalho, por sua vez, é mais recente, com intensificação dos estudos na década de 1980. Essa trata as características de composição dos setores de atividades econômicas, como melhores indicadores de distribuição e de níveis de renda, como determinantes da migração para uma determinada região (LIMA, 1980; BARROS; FRANCO; MENDONÇA, 2007). Nesse sentido, regiões com setores mais estruturados, com ocupações de melhor qualidade e que possuem melhores remunerações tendem a ser mais atrativas para a migração positivamente selecionada.

$\mathrm{Na}$ literatura econômica nacional não há consenso acerca dos fatores determinantes da seleção positiva migratória interna, apesar da relevante quantidade de autores que afirmam que os migrantes brasileiros são positivamente selecionados (FREGUGLIA, 2007; GAMA; MACHADO, 2014; RIGOTTI; CAMPOS; HADAD, 2017; CORREIA; OJIMA, 2017; SILVA FILHO et al., 2019; DE LIMA et al., 2019). Cabe, pois, considerar que estes estudos recorrem à base de dados diferentes, apesar de utilizarem métodos semelhantes para testar esta hipótese. Assim, os resultados podem apresentar diferenças quanto à hipótese de seleção migratória, haja vista que, em muitos estudos, tempo e distância da migração são fatores relevantes e não considerados pelos modelos.

Revisada a discussão teórica internacional e nacional acerca da hipótese de migração positivamente selecionada, que tratou dos determinantes da dinâmica migratória, apresentam-se na próxima seção os procedimentos metodológicos adotados para testar a hipótese para a região Centro-Oeste do Brasil no período intercensitário de 2000/2010. 


\section{Procedimentos Metodológicos}

Os dados utilizados neste estudo são oriundos dos Censos Demográficos do Brasil referentes aos anos de 2000 e 2010, conforme disponibilizados pelo Instituto Brasileiro de Geografia e Estatística (IBGE). A amostra é composta por indivíduos residentes na região Centro-Oeste com idade entre 18 e 60 anos que estivessem na situação de ocupados na semana de referência dos Censos Demográficos. O recorte etário se deu por duas razões: a primeira foi relacionada à decisão de migração ser de caráter individual, sendo possível àqueles que atingem a maioridade (18 anos) e podem inserir-se no mercado de trabalho sobre a proteção social instituída na legislação vigente; o segundo recorte se deu aos 60 anos, idade em que parte da força de trabalho migrante já atingiu a idade legal para a aposentadoria, sobretudo aqueles que auferem o benefício pelas prerrogativas da aposentadoria rural.

O Quadro 01 apresenta as variáveis selecionadas, dependentes e explicativas de controle empregadas no modelo. Ressalta-se que tais variáveis refletem aspectos da dinâmica socioeconômica e demográfica no território, e foram escolhidas com base na literatura que estuda o tema (MINCER, 1978; HECKMAN, 1979; FREGUGLIA, 2007; CUTILLO; CECCARELLI, 2012; GAMA; MACHADO, 2014; SILVA FILHO, 2017; SILVA; CAVALCANI; SILVA FILHO, 2019).

A variável dependente migra foi utilizada para testar a probabilidade de ser migrante dos indivíduos domiciliados no Centro-Oeste, a partir da estimação do primeiro estágio de Heckman. A variável dependente ln_rendatrab, por sua vez, foi utilizada para captar os diferenciais de rendimentos entre os migrantes por características socioeconômicas e demográficas a partir da estimação do segundo estágio de Heckman.

As variáveis independentes - explicativas de controle - foram utilizadas para controlar a discriminação. Barros, Franco e Mendonça (2007) explicam que o mercado de trabalho brasileiro é discriminador, especialmente quando se trata dos diferenciais de rendimentos do trabalho entre homens e mulheres, e brancos e não brancos, em desfavor do segundo grupo em ambas as comparações. 


\section{Quadro 1: Variáveis Dependentes e Explicativas de Controle dos Modelos.}

\begin{tabular}{|c|c|}
\hline Variável & Descrição \\
\hline \multicolumn{2}{|r|}{ Variáveis dependentes } \\
\hline Migra & $\begin{array}{l}\text { Varável dummy que é igual a } 1 \text { quando o indivíduo é migrante e } 0 \text { quando o } \\
\text { indivíduo é não migrante. }\end{array}$ \\
\hline Ln_rendatrab & $\begin{array}{l}\text { Variável contínua que representa o logaritmo neperiano da renda do trabalho } \\
\text { do indivíduo. }\end{array}$ \\
\hline \multicolumn{2}{|r|}{ Variáveis explicativas de controle } \\
\hline Sexo & $\begin{array}{l}\text { Variável dummy que é igual a } 1 \text { quando o indivíduo é do sexo masculino e } \\
\text { igual a } 0 \text { quando o indivíduo é do sexo feminino. }\end{array}$ \\
\hline Racacor (Branco) & $\begin{array}{l}\text { Variável dummy que é igual a } 1 \text { quando o indivíduo é branco e igual a } 0 \\
\text { quando o indivíduo é não branco. }\end{array}$ \\
\hline Idade & Variável contínua que representa a idade do indivíduo. \\
\hline Idade $^{2}$ & Variável idade elevada ao quadrado. \\
\hline Estadocivil & $\begin{array}{l}\text { Variável dummy que é igual a } 1 \text { quando o indivíduo é casado e igual a } 0 \\
\text { quando o indivíduo é solteiro. }\end{array}$ \\
\hline Chefedom & $\begin{array}{l}\text { Variável dummy que é igual a } 1 \text { quando o indivíduo é o chefe do domicílio } \\
\text { e igual a } 0 \text { caso contrário. }\end{array}$ \\
\hline Filho & $\begin{array}{l}\text { Variável dummy que é igual a } 1 \text { quando o indivíduo tem a posição de filho e } \\
\text { igual a } 0 \text { caso contrário. }\end{array}$ \\
\hline Seminstfundinc & $\begin{array}{l}\text { Variável dummy que é igual a } 1 \text { quando o indivíduo é sem instrução e/ou } \\
\text { possui o fundamental incompleto. }\end{array}$ \\
\hline Fundcompmedinc & $\begin{array}{l}\text { Variável dummy que é igual a } 1 \text { quando o indivíduo possui o fundamental } \\
\text { completo e/ou o médio incompleto. }\end{array}$ \\
\hline Medcompsupinc & $\begin{array}{l}\text { Variável dummy que é igual a } 1 \text { quando o indivíduo possui o ensino médio } \\
\text { completo e/ou o superior incompleto. }\end{array}$ \\
\hline Supcomp & $\begin{array}{l}\text { Variável dummy que é igual a } 1 \text { quando o indivíduo possui o ensino superior } \\
\text { completo. }\end{array}$ \\
\hline BRA & Variável dummy que é igual a 1 quando o indivíduo reside em Brasília. \\
\hline MS & $\begin{array}{l}\text { Variável dummy que é igual a } 1 \text { quando o indivíduo reside no estado do Mato } \\
\text { Grosso do Sul. }\end{array}$ \\
\hline MT & $\begin{array}{l}\text { Variável dummy que é igual a } 1 \text { quando o indivíduo reside no estado do Mato } \\
\text { Grosso. }\end{array}$ \\
\hline GO & $\begin{array}{l}\text { Variável dummy que é igual a } 1 \text { quando o indivíduo reside no estado do } \\
\text { Goiás. }\end{array}$ \\
\hline
\end{tabular}

Fonte: IBGE; elaborado pelos autores (2020).

As variáveis idade e idade (ao quadrado) são correntemente utilizadas na literatura que utiliza equações mincerianas para controlar o efeito da experiência e do ponto de inflexão quando se atinge uma idade elevada, tendendo-se à aposentadoria. Assim, esperase que a idade possua correlação positiva com a renda, enquanto a idade ao quadrado apresente um crescimento da renda positivo, mas menor, após ultrapassar o turning point.

Becker (1993) coloca o nível de instrução formal do indivíduo como um dos principais determinantes da probabilidade de migrar e do sucesso dessa migração - o 
segundo em termos de renda. Para verificar tal dinâmica, utilizar-se-á como proxy os anos de estudo dos indivíduos, categorizados por quatro faixas de formação, a saber: $i$ ) Sem instrução e/ou fundamental incompleto; $i i$ ) Fundamental completo e/ou médio incompleto; iii) Médio completo e/ou superior incompleto; e iv) Superior completo. Becker (1993) explica que quanto maior o nível de instrução, maior tende a ser a probabilidade de migração e maior tende a ser o retorno monetário.

Com o intuito de capturar a presença de um viés seletivo no processo migratório para a região Centro-Oeste no período 2000 - 2010, foi desenvolvido um modelo econométrico a partir de uma equação minceriana, nos moldes da encontrada em Santos Júnior et al. (2005), Gama e Machado (2014), Silva Filho (2017) e Silva Filho et al. (2019), como segue:

$$
\ln \left(w_{i}\right)=\alpha+\beta^{\prime} X_{i}+\phi M_{i}+u_{i}
$$

onde $w_{i}$ é a variável dependente que representa uma função de rendimento do trabalho do indivíduo $\mathrm{i} ; X_{i}$ expressa um vetor de variáveis socioeconômicas e demográficas observáveis explicativas de controle; $M_{i}$ corresponde a uma variável dummy de migração onde 1 é condicionado ao indivíduo migrante e 0 ao não migrante; $\alpha$ é a constante; $\beta$ é o coeficiente relacionado ao vetor de variáveis explicativas $X_{i}$; e $u_{i}$ o erro residual do modelo.

Se estimado por Mínimos Quadrados Ordinários (MQO) o coeficiente de $M_{i}$ pode ser viesado, sabendo-se que este método não permite controlar o viés de seleção amostral. Estimando-se por equações mincerianas, é possível que não se controle o viés de seleção e, assim sendo, poderá existir correlação entre o termo de erro e a dummy de migração. Assim, se (1) for estimado por MQO, e o resultado apresentar coeficiente $\phi$ positivo e estatisticamente significativo, não implica em seleção positiva migratória, necessariamente. Como solução, adotou-se o procedimento de Heckman (1979), em dois estágios.

Sendo a decisão de migração $M_{i}$ relacionada a características observáveis e não observáveis, estima-se que:

$$
M_{i}^{*}=\delta Z_{i}+\varepsilon_{i}
$$

onde, $Z_{i}$ comporta as características observáveis que podem ter relação com a decisão de migração. Nesta perspectiva, é possível que o indivíduo migre $(M=1)$ se $M^{*}>0$. Ou seja, sua probabilidade de migração pode estar relacionada com a probabilidade de auferir rendimentos do trabalho maior que zero.

Estimando por Heckman (1979), o primeiro estágio consiste em regredir o modelo (2) por meio de um Probit Univariado, conforme a equação (3), abaixo.

$$
P(M=1)=P\left(M^{*}>0\right)=P\left(\delta Z_{i}+\varepsilon_{i}>0\right)=P\left(\varepsilon_{i}>-\delta Z_{i}\right)
$$

sendo que $u_{i}$ e $\varepsilon_{i}$ são normalmente distribuídos, com média zero e correlação $\rho$, a esperança da equação (1) pode ser definida como em (4):

$$
E\left[\ln \left(w_{i}\right) \mid \varepsilon_{i}>-\delta Z_{i}\right]+u_{i}=\alpha+\beta^{\prime} X_{i}+\phi M_{i}+\delta \lambda_{i}\left(a_{\varepsilon}\right)+u_{i}
$$


onde,

$$
a_{\varepsilon}=-\delta Z_{i} / \sigma_{\varepsilon} \text { e } \lambda\left(a_{\varepsilon}\right)=\frac{\phi\left(\delta Z_{i} / \sigma_{\varepsilon}\right)}{\Phi\left(\delta Z_{i} / \sigma_{\varepsilon}\right)}
$$

sendo que $\lambda$ corresponde ao Inverso da Razão de Mills.

A partir da equação (5) é pertinente destacar que, segundo Greene (2003), se $\rho \neq 0$, inclui-se $\lambda$ na equação de interesse, uma vez que a ausência dele levaria a estimações viesadas. Na literatura dominante sobre o tema, é feita somente a análise da significância estatística do $\lambda$ e do sinal de $\rho$. Se for $\lambda$ estatisticamente significativo, confirma-se a importância da correção do viés de seleção. $\mathrm{O} \rho$ - sendo a correlação entre os componentes estocásticos das duas especificações de Heckman (1979) - indica se as variáveis não observáveis $\left(u_{i}\right.$ e $\left.\varepsilon_{i}\right)$ são positivamente ou negativamente correlacionadas com o primeiro e o segundo estágios do modelo de Heckman (VARTATIAN, 2015).

Depois de feita a devida correção, o segundo estágio do método de Heckman (1979) é estimado por MQO, conforme equação (6):

$$
\ln \left(w_{i}\right)=\alpha+\beta^{\prime} X_{i}+\phi M_{i}+\delta \lambda_{i}+u_{i}
$$

Se o coeficiente relacionado ao Inverso da Razão de Mills apresentar significância estatística, confirma-se a importância da correção do viés de seleção amostral. Enquanto $\rho$, é considerando como detentor de um conjunto de características não observáveis em que $\rho>0$ confirma que fatores não observáveis estão positivamente correlacionados com a decisão de migração (primeiro estágio), e podem também correlacionar-se aos diferenciais de rendimentos (segundo estágio). De forma análoga, um $\rho<0$ implica que os fatores não observáveis têm relação inversa com a probabilidade de migrar. Ou seja, os migrantes não são positivamente selecionados.

Apresentada a estratégia empírica adotada, a seção seguinte explora os principais resultados e a discussão dentro da literatura nacional e internacional.

\section{Resultados e Discussão}

\subsection{Dinâmica Socioeconômica e Demográfica do Centro-Oeste no Período 2000/2010}

A partir dos dados trabalhados dos Censos Demográficos do IBGE, o primeiro procedimento, exposto nessa seção, foi o de realizar uma caracterização socioeconômica e demográfica da população da região do Centro-Oeste do Brasil, nos anos de 2000 e 2010, no que tange aos diferenciais entre indivíduos migrantes e não migrantes. Tal caracterização possui, portanto, um objetivo estatístico descritivo. Além de conhecer o perfil dos migrantes e não migrantes no período, isso permite uma comparabilidade com trabalhos que estudaram outras localidades ${ }^{1}$, assim como uma análise mais acurada dos

\footnotetext{
${ }^{1}$ Conferir, por exemplo, Gama e Machado (2014) e Silva Filho et al. (2019), que estudaram o Brasil e o Rio Grande do Norte, respectivamente.
} 
resultados obtidos a partir dos procedimentos econométricos que serão expostos na seção posterior.

A formatação exposta dos dados (Tabela 1) mostra as participações - quando se trata de variáveis dummies - e as médias - quando consideradas variáveis contínuas. Como é possível perceber, a maioria é composta por homens, tanto migrantes quanto não migrantes, e apresentam redução no ano de 2010, comparativamente ao ano de 2000, diferentemente do que ocorre no Brasil. Muito embora os homens tenham maior probabilidade de serem migrantes, o valor do coeficiente da variável para o ano 2010 se eleva, conforme Silva Filho (2017). Ou seja, aumenta-se a participação das mulheres ocupadas na região Centro-Oeste do país. Esse resultado reforça as evidências de Barros, Franco e Mendonça (2007) para o mercado de trabalho brasileiro e evidenciam que ele é similar ao da região Centro-Oeste quanto ao seu caráter discriminador. Esse movimento é positivo e tende a potencializar a produtividade total da economia.

No caso da raça/cor, reduz-se a participação da população declarada branca na região, seja pelo maior acesso de não brancos ao mercado de trabalho, seja pela maior conscientização de autodeclaração de raça/cor nos últimos anos. Pelos resultados, a maioria dos ocupados na região, no ano de 2010, são não brancos, tanto migrantes quanto não migrantes. Tais aumentos na participação dos não brancos têm sido intensivamente estudados. Um argumento é que o maior esclarecimento e instrução têm elevado a autodeclaração de indivíduos não brancos. Ademais, a idade média da população migrante é inferior à da população não migrante nos dois anos em análises. Isso ocorre porque, como explicado por Mincer (1978), os indivíduos sem dependentes tendem a ser mais favoráveis ao risco, e, portanto, são mais propícios a buscarem remuneração superior a partir da migração, como argumentam Massey (1993) e White (2016), que tratam a migração como investimento.

No que se refere ao estado civil, em ambos os anos a participação relativa dos casados é inferior àquela em outra condição matrimonial. Isto é, pode-se estar associado à conclusão de que a migração é mais intensa nos municípios para pessoas jovens e solteiras (SILVA FILHO et al., 2019). Registra-se redução relativa da população casada ocupada, tanto migrante quanto não migrante na região, quando comparado o ano de 2010 ao ano 2000. Tal evidência revela que as pessoas estão, em média, contraindo matrimônio mais tardiamente. Adicionalmente, pode-se destacar que a participação relativa de pessoas que se declararam chefes de domicílios é estatisticamente igual tanto para migrantes quanto para não migrantes e permanece praticamente constante ao longo da década de 2000. 
Tabela 1: Caracterização Socioeconômica e Demográfica da População Ocupada no Centro-Oeste - 2000/2010

\begin{tabular}{l|c|c|c|c}
\hline \multirow{2}{*}{ Variáveis } & \multicolumn{2}{|c|}{ 2000 } & \multicolumn{2}{c}{ 2010 } \\
\cline { 2 - 5 } & Migrante & Não Migrante & Migrante & Não Migrante \\
\hline Sexo & 0,66 & 0,67 & 0,62 & 0,62 \\
\hline Racacor (Branco) & 0,48 & 0,50 & 0,42 & 0,44 \\
\hline Idade & 33,07 & 35,46 & 35,16 & 38,57 \\
\hline Idade $^{2}$ & 1204,82 & 1382,37 & 1356,28 & 1618,44 \\
\hline Estadocivil $_{\text {Chefedom }}$ & 0,43 & 0,48 & 0,37 & 0,44 \\
\hline Filho & 0,55 & 0,55 & 0,54 & 0,54 \\
\hline Seminstfundinc & 0,11 & 0,20 & 0,07 & 0,15 \\
\hline Fundcompmedinc & 0,65 & 0,64 & 0,46 & 0,48 \\
\hline Medcompsupinc & 0,16 & 0,16 & 0,20 & 0,18 \\
\hline Supcomp & 0,16 & 0,18 & 0,25 & 0,25 \\
\hline BRA & 0,02 & 0,02 & 0,10 & 0,08 \\
\hline MS & 0,10 & 0,11 & 0,05 & 0,06 \\
\hline MT & 0,14 & 0,17 & 0,17 & 0,18 \\
\hline GO & 0,30 & 0,23 & 0,30 & 0,25 \\
\hline Rendatrab & 0,46 & 0,48 & 0,49 & 0,51 \\
\hline Ln_rendatrab & $1.249,90$ & $1.411,77$ & $1.422,25$ & $1.385,74$ \\
\hline \hline
\end{tabular}

Fonte: IBGE, elaboração dos autores a partir de dados dos Censos Demográficos do Brasil (2020).

Por outro lado, há redução da participação daqueles que se declararam filhos, sendo que esta característica foi relativamente inferior para os migrantes em comparação com os não migrantes. Logo, infere-se que assim como tem reduzido o número de casados, tem-se reduzido também a quantidade de filhos, que reflete a natalidade menor observada nas décadas de 1990 e 2000 . Tais estatísticas levantam evidências de que os argumentos de Mincer (1978), no que tange a influência das características familiares na decisão de migrar, e de Massey (1993) e White (2016), quanto ao tratamento da migração como um investimento que tem risco, são pertinentes para o caso da região Centro-Oeste do Brasil no período em análise.

No que se refere à escolaridade, é possível constatar, diante dos registros, a redução relativa da participação daqueles sem instrução ou com ensino fundamental incompleto, tanto entre migrantes quanto não migrantes no ano de 2010, comparativamente ao ano 2000. No ano 2000, mais de $60 \%$ de ambos os grupos estavam em situação de baixa escolaridade formal. Já no ano de $2010,46 \%$ da força de trabalho migrante e $48 \%$ da não migrante se declarou sem instrução ou com ensino fundamental incompleto. Cabe, portanto, registrar que ao término da primeira década do século XXI parte substancialmente elevada da força de trabalho ocupada na região Centro-Oeste tem baixa escolaridade (sem instrução ou fundamental incompleto).

Foi possível constatar que ao longo da década de 2000 houve um relativo aumento da participação de indivíduos com ensino médio completo e superior incompleto na força 
de trabalho ocupada do Centro-Oeste brasileiro. Em ambos os grupos foi registrado o total de $25 \%$ de seus ocupados com estes níveis de escolaridade. Ademais, é pertinente destacar a elevação expressiva daqueles com ensino superior completo. No ano 2000 , somente $2 \%$ dos ocupados migrantes, e o mesmo percentual dos não migrantes, tinham curso superior completo na região Centro-Oeste. Em 2010 a participação dos migrantes com curso superior chega a $10 \%$ e a dos não migrantes atinge $8 \%$ na região. Tal evidência corrobora os argumentos de Becker (1993), que tem o nível de instrução formal como um dos principais determinantes da migração e seu sucesso. Vale destacar, segundo Santos Junior et al. (2005), que quanto mais elevado o nível de escolaridade, maior a média salarial dos indivíduos, e que os saltos salariais são cada vez maiores a cada mudança de nível de instrução. Esse fator corrobora a teoria do capital humano, postulando que quanto maior o nível de investimento em educação e qualificação, maior deverá ser a remuneração dos indivíduos.

No que se refere à distribuição espacial da população, como visto na tabela acima, a dinâmica populacional no Centro-Oeste se deu de tal forma que Brasília reduziu sua participação relativa tanto de migrantes quanto de não migrantes ocupados no ano 2010 , comparativamente ao ano 2000. O Estado de Goiás apresentou aumento da participação de pessoas com idade entre 18 e 60 anos ocupadas, tanto migrantes quanto não migrantes, saindo de $46 \%$ para $49 \%$ daqueles e de $49 \%$ para $51 \%$ destes, em 2010. Mato Grosso do Sul também registrou aumento de migrantes ocupados de $14 \%$ para $17 \%$ do total da região, sendo que a população ocupada não migrante registrou $17 \%$ no primeiro e $18 \%$ no último ano em observação. Já o Estado do Mato Grosso, que ocupa a segunda posição no ranking ocupacional da região, registrou $30 \%$ dos migrantes ocupados e manteve sua participação relativa em 2010, sendo que a população não migrante ocupada era inferior à migrante (no primeiro ano, 23\%; no segundo, 25\%). Infere-se que a migração tem contribuído para elevar o capital humano e o desenvolvimento dessas regiões, como teorizaram Lee (1966), Ravenstein (1980) e Bastia e Skeldon (2019), haja vista que estes possuem características vantajosas, tanto observáveis quanto não observáveis (BORJAS, 1987; DUSTMANN; GLITZ, 2011).

No que se refere à renda do trabalho, os migrantes, no ano 2000, ganhavam em média $\mathrm{R} \$ 1.249,90$ e os não migrantes auferiam rendimentos médios do trabalho de $\mathrm{R} \$ 1.411,77$. Destarte, a renda média do trabalho dos ocupados migrantes era inferior àquela auferida pelos nativos. No ano de 2010 eleva-se substancialmente a renda média dos migrantes e ela supera a renda média dos nativos, que também registrou redução. Neste ano, a renda média dos migrantes era de $\mathrm{R} \$ 1.422,25$ e a dos não migrantes reduziu-se à $\mathrm{R} \$ 1.385,74$. Essa evidência reforça que os migrantes contribuem para o desenvolvimento regional, ampliando o estoque de conhecimento e experiências, como colocam Johnson e Salt (2018), compreendendo que os maiores rendimentos do trabalho sao resultados de mais investimento em qualificação e capital humano (LEE, 1966; RAVENSTEIN, 1980; BECKER, 1993; SANTOS JUNIOR et al., 2005; BASTIA e SKELDON, 2019), bem como de atributos não observáveis que afetam a decisão de migração e também podem impactar 
nos rendimentos do trabalho (BORJAS, 1987; DUSTMANN; GLITZ, 2011; SILVA FILHO, 2017).

Com relação à distribuição espacial da população ocupada com idade entre 18 e 60 anos, tanto o mapa à esquerda (ano 2000) como o mapa à direita (ano 2010), na Figura 1, mostram que há ganhos líquidos (maior entrada que saída) da força de trabalho nas capitais dos Estados e nos municípios economicamente desenvolvidos da região. Adicionalmente, destaca-se que, no ano 2000, os municípios do Estado do Mato Grosso, em sua maioria, registraram saldos líquidos positivo de vagas, e a distribuição espacial da população não se configurou num padrão de concentração, mas de dispersão ao longo de todo o Estado. Em 2010, mesmo com a redução dos fluxos, este Estado mantém entrada líquida positiva na maioria de seus municípios.

Figura 1: Saldo Migratório da Força de Trabalho Ocupada nos Municípios do Centro-Oeste - 2000/2010
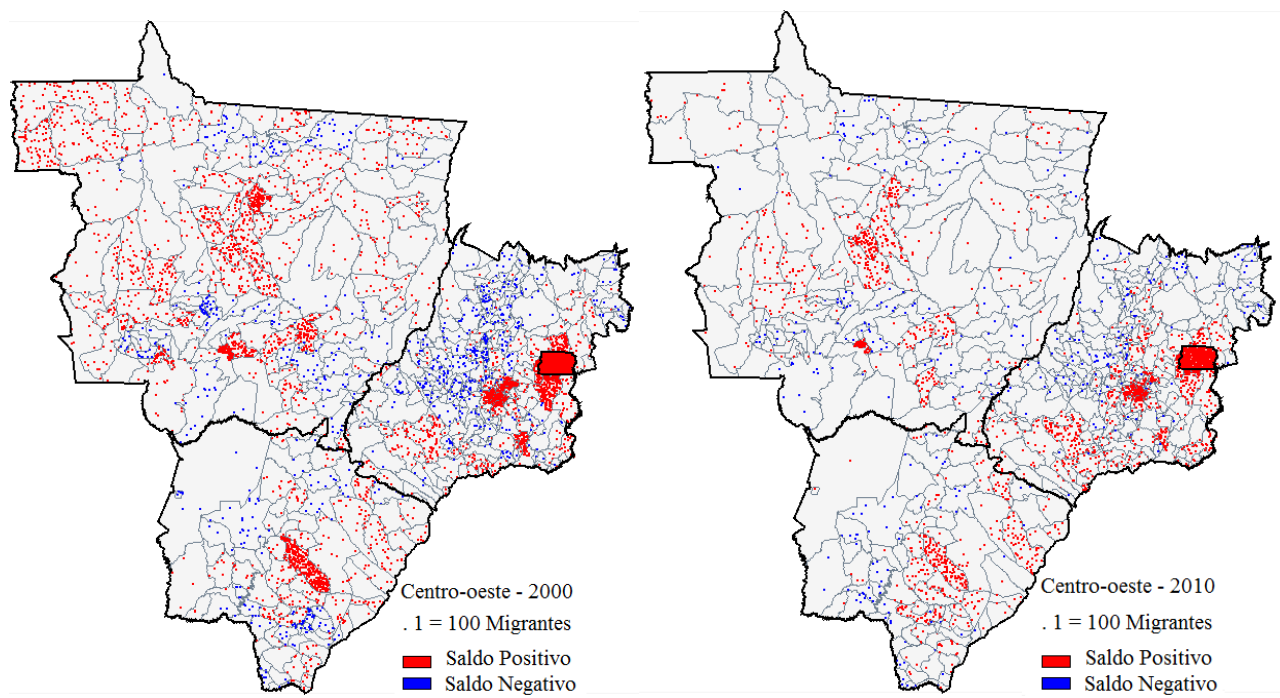

Fonte: IBGE, elaboração dos autores a partir de dados dos Censos Demográficos (2020).

Em 2010 é possível observar que as áreas atrativas, em sua grande maioria, mantiveram o ingresso líquido positivo de força de trabalho. O que se reduziu, de fato, foram os fluxos de ocupados com idade na faixa estabelecida nesta análise. Porém, a Capital Federal e as capitais dos demais Estados do Centro-Oeste mostraram-se atrativas ao ingresso da força de trabalho migrante na década de 2000. Isso já era esperado, uma vez que a busca por melhores condições de vida fazem com que os indivíduos migrantes, mais determinados e persistentes que indivíduos não migrantes com características socioeconômicas observáveis semelhantes, migrem para essas cidades (BORJAS, 1987; DUSTAMANN; GLITZ, 2011; CUTILLO; CECCARELLI, 2012).

$\mathrm{Na}$ Figura 2, as informações plotadas referem-se às faixas salariais da força de trabalho ocupada nos municípios da região Centro-Oeste. A cor mais escura (vermelho) 
são os municípios que tinham remuneração média superior a dois salários mínimos. É possível perceber que são aqueles de maior dinamismo econômico que concentram também as melhores remunerações médias à força de trabalho e estão localizados, em sua maioria, ao Norte da região. Os demais Estados apresentam poucos municípios com remuneração média na faixa de mais de dois salários mínimos. Em toda a região, registra-se substancial número de municípios com remuneração média entre 1,5 e até 2,0 salários mínimos. Observa-se redução de disparidades intrarregionais, suavizando a faixa de remuneração da força de trabalho tanto de forma intra quanto de forma interestadual, entre 2000 e 2010 . No entanto, o Estado de Mato Grosso aponta uma elevação na disparidade de renda, diferentemente dos demais Estados da região.

\section{Figura 2: Faixa de Remuneração da Força de Trabalho Ocupada nos Municípios do Centro-Oeste - 2000/2010}
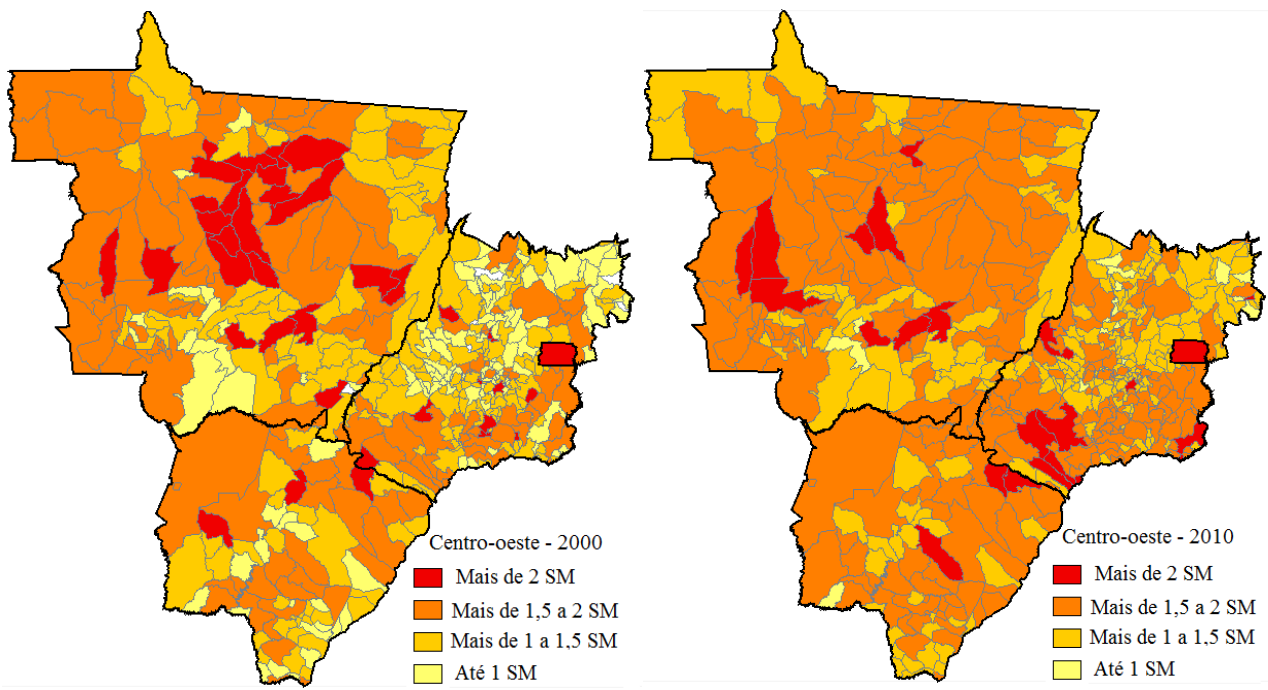

Fonte: IBGE, elaboração dos autores a partir de dados dos Censos Demográficos (2020).

No ano de 2010 é possível perceber uma redução dos municípios na faixa de remuneração média superior a dois salários mínimos e a elevação substancial do número de municípios com remuneração média entre 1,5 e 2,0 salários mínimos. No Mato Grosso do Sul, somente 3 municípios estavam na faixa de remuneração média superior a dois salários mínimos. Municípios no sul do Goiás e alguns municípios dispersos no território mato-grossense registraram a melhor faixa de remuneração. De modo geral, a maioria dos municípios do Goiás e do Mato Grosso do Sul registrou remuneração do trabalho na faixa mais baixa de até 1 salário mínimo, confirmando modificações fundamentais nos aspectos socioeconômicos das áreas historicamente mais pobres de cada estado, não só em virtude do impulso promovido pela expansão da fronteira agrícola, mas também da ocupação urbana. 


\subsection{Análise de Diferenciais de Rendimentos entre os Migrantes por Características Socioeconômicas e Demográficas - 2000/2010}

Nessa seção, a partir das estimações econométricas propostas na abordagem empírica - seção dois - são apresentados os resultados e as discussões decorrentes. A partir da estimação do primeiro estágio de Heckman, que permite captar a probabilidade de migração, são obtidos os resultados reportados na Tabela 2. E a partir da estimação do segundo estágio de Heckman, que permite captar os diferenciais de rendimentos entre os migrantes por características socioeconômicas e demográficas, são obtidos os resultados apresentados na Tabela 3 .

Pelos resultados da Tabela 2, observa-se que o indivíduo migrante possuía maior probabilidade de ser homem, comparativamente às mulheres, tanto no primeiro quanto no último ano. Porém, pode-se observar a redução dessa probabilidade no ano de 2010, fato que pode ser uma consequência da tendência geral de aumento da participação das mulheres no mercado de trabalho (BARROS; FRANCO; MENDONÇA, 2007). Os brancos, por sua vez, tinham maior probabilidade de serem migrantes intermunicipais no Centro-Oeste no primeiro ano e reduziram a probabilidade no segundo ano. Como abordado anteriormente, tal movimento pode estar relacionado tanto à redução do preconceito quanto à conscientização da população sobre autodeclaração (SILVA FILHO, 2017). Por sua vez, aqueles que se declaram brancos, no Brasil, apresentam proporção consideravelmente mais elevada entre os ocupados em comparação à população total.

Em relação à idade, a probabilidade se reduz com o aumento dessa, ou seja, à medida que a idade vai avançando a probabilidade de ser migrante é reduzida. Isso significa dizer que a população migrante é, em sua maioria, composta por jovens em busca de oportunidades de trabalho. Tal resultado é similar aos obtidos por Rodrigues et al. (2015) e Rigotti et al. (2017), e reforça a argumentação de Mincer (1978), dado que indivíduos mais novos possuem probabilidade menor de ter familiares dependentes.

Com relação à dinâmica entre escolaridade e probabilidade de migração, declarantes sem instrução ou com ensino fundamental incompleto apresentaram maior probabilidade em ambos os anos, em relação aos indivíduos com ensino fundamental completo e médio incompleto, e indivíduos com ensino médio completo e superior incompleto. Por outro lado, a probabilidade de estar ocupado e ser migrante é maior para os indivíduos com curso superior completo e se elevou no ano de 2010, comparativamente ao ano 2000. Dessa forma, os argumentos de Cutillo e Ceccarelli (2012) de que uma maior educação aumenta a probabilidade de migração é corroborada apenas no caso do ensino superior, para a região Centro-Oeste. 
Tabela 2: Estimativas da Probabilidade de Migração para os Municípios do CentroOeste nos Censos de 2000 e de 2010

\begin{tabular}{|c|c|c|}
\hline \multirow{2}{*}{ Variáveis } & \multicolumn{2}{|c|}{ Variável Dependente = Migra } \\
\hline & 2000 & 2010 \\
\hline Constant & $\begin{array}{c}0,117 * * * \\
(0,025) \\
\end{array}$ & $\begin{array}{c}-0,173 * * * \\
(0,050)\end{array}$ \\
\hline Sexo (Masculino) & $\begin{array}{c}0,105^{* * *} \\
(0,005)\end{array}$ & $\begin{array}{c}0,076^{* * *} \\
(0,009)\end{array}$ \\
\hline Racacor (Branco) & $\begin{array}{c}0,006 \\
(0,004) \\
\end{array}$ & $\begin{array}{c}-0,011 \\
(0,008) \\
\end{array}$ \\
\hline Idade & $\begin{array}{c}-0,041 * * * \\
(0,001)\end{array}$ & $\begin{array}{c}-0,038 * * * \\
(0,003)\end{array}$ \\
\hline Idade $^{2}$ & $\begin{array}{c}0,0003 * * * \\
(0,00002)\end{array}$ & $\begin{array}{l}0,0002 * * * \\
(0,00003)\end{array}$ \\
\hline Fundcompmedinc & $\begin{array}{c}-0,019 * * * \\
(0,006)\end{array}$ & $\begin{array}{c}-0,002 \\
(0,011) \\
\end{array}$ \\
\hline Medcompsupinc & $\begin{array}{c}-0,035 * * * \\
(0,006)\end{array}$ & $\begin{array}{c}-0,022 * * \\
(0,010)\end{array}$ \\
\hline Supcomp & $\begin{array}{c}0,080 * * * \\
(0,015) \\
\end{array}$ & $\begin{array}{c}0,170 * * * \\
(0,015) \\
\end{array}$ \\
\hline Chefedom & $\begin{array}{c}-0,151 * * * \\
(0,006)\end{array}$ & $\begin{array}{c}-0,070 * * * \\
(0,009)\end{array}$ \\
\hline Estadocivil & $\begin{array}{c}-0,141 * * * \\
(0,005)\end{array}$ & $\begin{array}{c}-0,173 * * * \\
(0,009)\end{array}$ \\
\hline Filho & $\begin{array}{c}-0,792 * * * \\
(0,008) \\
\end{array}$ & $\begin{array}{c}-0,780 * * * \\
(0,016) \\
\end{array}$ \\
\hline MS & $\begin{array}{c}0,026 * * * \\
(0,008)\end{array}$ & $\begin{array}{c}0,188 * * * \\
(0,020)\end{array}$ \\
\hline MT & $\begin{array}{c}0,277 * * * \\
(0,008) \\
\end{array}$ & $\begin{array}{c}0,302 * * * \\
(0,019)\end{array}$ \\
\hline GO & $\begin{array}{c}0,108 * * * \\
(0,007) \\
\end{array}$ & $\begin{array}{c}0,195 * * * \\
(0,019) \\
\end{array}$ \\
\hline Observações & 537,794 & 170,158 \\
\hline
\end{tabular}

Fonte: IBGE, elaboração dos autores a partir de dados dos Censos Demográficos do Brasil (2020).

Note: $* \mathrm{p}<0,1 ; * * \mathrm{p}<0,05 ; * * * \mathrm{p}<0,01$

Os dados da Tabela 2 indicam que a probabilidade de ser migrante é menor para chefes de domicílios, casados e os que ocupam a posição de filho nos domicílios, em detrimento dos que ocupam outras posições e que estão em outros status maritais. Essas evidências corroboram a argumentação de Mincer (1978) para o caso em análise, sendo, portanto, as variáveis familiares importantes determinantes da migração dos indivíduos.

No que tange à localização, tendo o Distrito Federal como referência, a probabilidade de residir em qualquer um dos Estados do Centro-Oeste e ser migrante é maior no primeiro e no último ano em análise. Adicionalmente, a probabilidade de residir no Estado do Mato 
Grosso e ser migrante é superior àquela registrada nos demais Estados da Região, comparativamente a um residente em Brasília. Nesse sentido, como colocado por Borjas (1987) e Dustmann e Glitz (2011), essas regiões podem ser beneficiadas pela migração, dada pelas suas características potenciais intrínsecas, como determinação e persistência.

$\mathrm{Na}$ Tabela 3 (segundo estágio de Heckman) cabe chamar atenção para o fato de que a hipótese de seleção positiva migratória nos municípios do Centro-Oeste foi confirmada, uma vez que a significância estatística do Inverso da Razão de Mills ocorreu a menos de $1 \%$ de significância. Ou seja, características não observáveis afetam a decisão de migração dos indivíduos. Todas as variáveis apresentaram significância estatística a $1 \%$.

Conforme exposto na Tabela 3, os homens migrantes ganham em média mais que as mulheres em ambos os anos. Chama atenção o crescimento das disparidades de rendimentos entre homens e mulheres migrantes no Centro-Oeste, uma vez que em 2000, um homem auferia rendimento aproximadamente 50\% superior a uma mulher na mesma região. No ano de 2010, esta disparidade se eleva para 55\%. A explicação parcial das diferenças salariais elevadas entre homens e mulheres pode vir de: i) forte participação do agronegócio e das atividades agropecuárias na região, guetos predominantemente masculinos na ocupação da força de trabalho; ii) atividades nestes setores serem, em essência, remuneradas com parcela fixa e parcela variável pela produtividade, sendo o esforço físico importante determinante da produtividade e da renda.

No que se refere à raça/cor, no ano 2000, os migrantes autodeclarados brancos auferiam 21\% a mais nos rendimentos do trabalho que um migrante não branco. Em 2010 reduz-se este gap para $17 \%$. As disparidades salariais ainda são elevadas quando se analisa por raça/cor a população migrante no Centro-Oeste. No que se refere à idade, um ano a mais confere retorno superior aos migrantes, em média, de $6 \%$ e $7 \%$, no primeiro e no último ano, respectivamente. Além disso, os retornos crescem, mas de maneira decrescente com a idade.

No que se refere aos retornos à educação, as evidências teóricas de Becker (1993) são corroboradas para o Centro-Oeste. Cabe ressaltar que todas as faixas de escolaridade elevam os rendimentos do trabalho em comparação aos menos escolarizados (sem instrução ou ensino fundamental incompleto - categoria de referência). Ademais, avançar de uma faixa de escolaridade para a outra proporciona melhores retornos salariais no mercado de trabalho para os migrantes mais escolarizados em detrimento daqueles com menor instrução formal, corroborando resultados de outros estudos para o Brasil e ou regiões (FREGUGLIA, 2007; GAMA; MACHADO, 2014; SILVA FILHO, 2017; DE LIMA; SILVA FILHO, CAVALCANTI 2019). Os que têm curso superior de formação ganham substancialmente mais que aqueles menos instruídos em ambos os anos, apesar de se registrar redução dos coeficientes de um ano para o outro, o que sugere que os retornos salariais à educação reduzem ao longo do tempo. 


\section{Tabela 3: Estimativa do Segundo Estágio de Heckman sobre a Determinação da Renda do Trabalho dos Migrantes no Centro-Oeste - 2000/2010}

\begin{tabular}{|c|c|c|}
\hline \multirow{2}{*}{ Variáveis } & \multicolumn{2}{|c|}{ Variável Dependente = ln_rendatrab } \\
\hline & 2000 & 2010 \\
\hline Constante & $\begin{array}{c}4,716 * * * \\
(0,035)\end{array}$ & $\begin{array}{c}4,951 * * * \\
(0,078)\end{array}$ \\
\hline Sexo & $\begin{array}{c}0,503 * * * \\
(0,007)\end{array}$ & $\begin{array}{c}0,558 * * * \\
(0,012)\end{array}$ \\
\hline Racacor & $\begin{array}{c}0,210 * * * \\
(0,006)\end{array}$ & $\begin{array}{c}0,165^{* * * *} \\
(0,011)\end{array}$ \\
\hline Idade & $\begin{array}{c}0,064 * * * \\
(0,002)\end{array}$ & $\begin{array}{c}0,067 * * * \\
(0,003)\end{array}$ \\
\hline Idade $^{2}$ & $\begin{array}{l}-0,001 * * * \\
(0,00002)\end{array}$ & $\begin{array}{l}-0,001 * * * \\
(0,00004)\end{array}$ \\
\hline Fundcompmedinc & $\begin{array}{c}0,390 * * * \\
(0,008) \\
\end{array}$ & $\begin{array}{c}0,258 * * * \\
(0,015) \\
\end{array}$ \\
\hline Medcompsupinc & $\begin{array}{c}0,887 * * * \\
(0,008) \\
\end{array}$ & $\begin{array}{c}0,532 * * * \\
(0,014) \\
\end{array}$ \\
\hline Supcomp & $\begin{array}{c}1,799 * * * \\
(0,019) \\
\end{array}$ & $\begin{array}{c}1,289 * * * \\
(0,020)\end{array}$ \\
\hline Chefedom & $\begin{array}{c}0,215^{* * *} \\
(0,007)\end{array}$ & $\begin{array}{c}0,110 * * * \\
(0,012)\end{array}$ \\
\hline Estadocivil & $\begin{array}{c}0,127 * * * \\
(0,006)\end{array}$ & $\begin{array}{c}0,126 * * * \\
(0,012)\end{array}$ \\
\hline MS & $\begin{array}{c}-0,329 * * * \\
(0,011)\end{array}$ & $\begin{array}{c}-0,260 * * * \\
(0,028)\end{array}$ \\
\hline MT & $\begin{array}{c}-0,097 * * * \\
(0,010) \\
\end{array}$ & $\begin{array}{c}-0,160 * * * \\
(0,028) \\
\end{array}$ \\
\hline GO & $\begin{array}{c}-0,263 * * * \\
(0,010) \\
\end{array}$ & $\begin{array}{c}-0,215 * * * \\
(0,027) \\
\end{array}$ \\
\hline Observações & 537,794 & 170,158 \\
\hline rho & $-0,138$ & $-0,180$ \\
\hline Inverse Mills Ratio & $\begin{array}{c}-0,113 * * * \\
(0,016)\end{array}$ & $\begin{array}{c}-0,141 * * * \\
(0,036)\end{array}$ \\
\hline
\end{tabular}

Fonte: elaboração dos autores a partir de dados dos Censos Demográficos do Brasil (2020). Note: $* \mathrm{p}<0,1 ; * * \mathrm{p}<0,05 ; * * * \mathrm{p}<0,01$.

Ademais, apesar de a probabilidade de migração dos chefes de domicílios e dos casados ser menor, comparativamente aos seus pares (Tabela 2), corroborando os argumentos de Mincer (1978) para o caso da região Centro-Oeste do Brasil, estes quando migram apresentam retorno salarial superior aos seus pares migrantes. Assim, é possível ver que, em 2000 e em 2010, os chefes de domicílios auferiam, em média, 22\% e 11\% a mais que seus comparativos, respectivamente. Já os casados tinham retorno salarial 13\%, em média, mais elevado que seus comparativos no primeiro e no último ano. Esse maior 
retorno está relacionado ao fato de que indivíduos que possuem participação mais importante na família - com dependentes, por exemplo - tendem a assumir menos riscos na migração e, portanto, exigem um retorno maior. Já os indivíduos independentes, com um vínculo de dependência menor em relação aos demais componentes da família tendem a assumir menos riscos e exigir menor retorno monetário pelo aumento do risco (HARRIS; TODARO, 1970; MINCER, 1978).

Com relação ao componente regional, os migrantes intermunicipais de todos os Estados da região Centro-Oeste auferiam rendimentos menores que um migrante em Brasília. As maiores disparidades salariais em relação à categoria de referência foram registradas no Estado do Mato Grosso do Sul, alcançando 33\% e 26\% em 2000 e em 2010, respectivamente. Ou seja, um migrante intermunicipal ganhava $33 \%$ menos que um migrante em Brasília no ano 2000, e $26 \%$ menos no ano de 2010. Segundo Silva Filho, Alves e Silva Júnior (2019), no Mato Grosso do Sul observa-se um aumento dos municípios com piora no quadro de pobreza domiciliar do sul do Estado, implicando em elevação dos diferenciais de renda.

Em Goiás, a disparidade era de $26 \%$ no primeiro ano, reduzindo-se para $22 \%$ em 2010. Para além do exercício espacial feito na Figura 2, a estimativa do segundo estágio de Heckman evidencia que o Estado do Mato Grosso foi o único a registrar elevação da disparidade no ano de 2010, comparativamente ao ano 2000. A disparidade de renda de um migrante intermunicipal deste Estado era de $10 \%$ a menos que um migrante intermunicipal em Brasília. Em 2010, eleva-se para 16\% essa diferença de renda, apesar de ser a menor entre os Estados da região Centro-Oeste, com referência à Capital Federal.

De modo geral, foi confirmada a hipótese de seleção positiva migratória nos municípios do Centro-Oeste que orientou o estudo. Os resultados se mostraram precisos e significantes no comparativo entre os dois anos, demonstrando uma disparidade de renda intermunicipal elevada, considerando Brasília. Posto isto, a seção seguinte tece algumas considerações finais do estudo em questão.

\section{Considerações Finais}

Este artigo partiu do objetivo de testar a hipótese de seleção migratória intermunicipal na região Centro-Oeste do Brasil, motivado pelo processo de povoamento ocorrido recentemente, impulsionado fortemente pela expansão da fronteira agrícola e ocupação urbana. Como abordagem empírica, a partir de dados cross-section de migração intermunicipal do Censo Demográfico do IBGE dos anos 2000 e 2010, adotou-se dois procedimentos: i) realizou-se uma caracterização socioeconômica e demográfica da região, comparando-se o perfil dos migrantes com o dos não migrantes; ii) empregou-se um modelo econométrico construído a partir de uma equação minceriana e estimado com o procedimento de seleção amostral de Heckman, que habilitou uma análise dos retornos monetários às características socioeconômicas e demográficas.

A caracterização revelou mudanças significativas e importantes de 2000 para 2010. Destacam-se quatro temas bastante debatidos: i) o aumento da participação das mulheres; 
ii) aumento da participação dos não brancos, sem distinção significativa entre migrantes e não migrantes; iii) o aumento do nível de educação, sendo que os migrantes possuem uma média levemente maior, especialmente quando se trata de ensino superior completo; iv) e o aumento da renda média dos migrantes frente a uma redução da renda média dos não migrantes. Os resultados das três primeiras dimensões, sexo, cor e educação indicam que a região tem acompanhado a tendência nacional, que está ocorrendo por diversos fatores, como redução do preconceito e maiores oportunidades, via conscientização e políticas públicas. Em relação à última dimensão, o rendimento médio, as evidências sugerem que os migrantes, além de possuírem níveis de educação maiores, podem possuir atributos que não são observáveis e que os favorecem frente aos não migrantes.

Aprofundou-se a investigação com o arcabouço econométrico e inferiu-se que existiu uma maior probabilidade de o migrante intermunicipal ser homem e não branco, reduzindo-se a probabilidade com o aumento de idade, e aumentando-se a probabilidade quando o indivíduo tem nível superior. Além disso, a probabilidade de ser migrante é menor para chefes de domicílios, casados e para os que ocupam a posição de filho. Esses resultados se alinham às evidências empíricas obtidas para outros recortes geográficos e que foram consultadas. Já em relação à localização, tem-se que os indivíduos residentes em Brasília são menos propensos a migrar, comparativamente aos Estados do Mato Grosso do Sul, Mato Grosso e Goiás. Isso pode estar relacionado ao fato de Brasília oferecer oportunidades, principalmente no âmbito do setor público e atividades correlatas, que desencorajam e não justificam a migração para outras regiões. Este argumento é fortalecido pela constatação de que os migrantes intermunicipais de todos os Estados da região CentroOeste auferiam rendimentos menores que um migrante em Brasília.

Por fim, as evidências revelaram que apesar da redução das desigualdades de participação por sexo e cor, os homens migrantes continuam ganhando em média mais que as mulheres, tendo a diferença, inclusive, aumentada no período. Já os migrantes brancos continuam com rendimento significativamente maior que os não brancos, mesmo com a redução da diferença no período. Portanto, o retorno às características não observáveis dos migrantes não superam as desigualdades presentes nessas dimensões, na região. Quanto à educação, apesar de todas as faixas de escolaridade elevarem os rendimentos com a progressão nos anos de estudo, o avanço de uma faixa de escolaridade superior proporciona ganhos mais que proporcionais aos migrantes. Além disso, os chefes de domicílios e os casados, quando migram apresentam retorno salarial superior aos seus pares migrantes. Tal resultado está alinhado à literatura e é justificado pelo fato de que esse perfil de migrante exige um prêmio maior ao risco de migrar.

A partir dessas evidências empíricas, chega-se à conclusão de que os indivíduos migrantes intermunicipais na região Centro-Oeste do Brasil no período intercensitário 2000-2010, são positivamente selecionados, com características produtivas não observáveis que afetam a decisão de migração. Apesar da robustez dos dados do Censo Demográfico do IBGE e da metodologia adotada, a restrição quantitativa do período em análise é uma limitação que pode esconder algum viés e que é uma oportunidade de ampliação e avanço da pesquisa em trabalhos posteriores. 


\section{Referências Bibliográficas}

BARROS, R. P.; FRANCO, S.; MENDONÇA, R. Discriminação e Segmentação no Mercado de Trabalho e Desigualdade de Renda no Brasil. Texto para Discussão IPEA, Rio de Janeiro, n.1288, 2007.

Disponível em: http://repositorio.ipea.gov.br/bitstream/11058/1842/1/TD_1288.pdf. Acessado em julho de 2019.

BASTIA, T.; SKELDON, R. Routledge Handbook of Migration and Development. New York: Routledge, 2019.

BECKER, G. S. Human Capital: a theoretical and empirical analysis, with special reference to education. University of Chicago press, 1993.

BORJAS, G. J. Economic Theory and International Migration. International Migration Review, v. 23, n. 3, p. 457-485, 1989. DOI: https://doi.org/10.2307/2546424.

BORJAS, G. J. Self-selection and the Earnings of Immigrants. The American Economic Review, v.77, n.4, p.531-53, 1987.

BRASIL, Instituto Brasileiro de Geografia e Estatística - IBGE, Censos Demográficos, 2000, 2010.

CORREIA, I. A.; OJIMA, R. Migração e Seletividade no Estado do Espírito Santo e na Região Metropolitana da Grande Vitória: Considerações a partir do censo demográfico de 2010. Geografares, Julho-Dezembro, 2017. DOI: https://doi.org/10.7147/GEO24.17174.

CUTILLO, A.; CECCARELLI, C. The Internal Relocation Premium: are migrants positively or negatively selected? Evidence from Italy. Journal of Applied Statistics, v. 39, n. 6, p. 1263-1278, 2012. DOI: https://doi.org/10.1080/02664763.2011.644529.

DE LIMA, Y. C.; FILHO, L. A.; CAVALCANTI, D. M. Migração, Seleção e Diferenciais de Renda na região Norte do Brasil em 2010. Revista Brasileira de Estudos Regionais e Urbanos, 13(1), 141-160, 2019.

DAO T. H.; DOCQUIER, F.; PARSONS, C.; PERI, G. Migration and development: Dissecting the Anatomy of the Mobility Transition. Journal of Development Economics, v. 132, p. 88-101, 2018. DOI: https://doi.org/10.1016/j.jdeveco.2017.12.003.

DUSTMANN, C.; GLITZ, A. Migration and Education. CReAM, Centre of Research and Analysis of Migration, Discussion Paper Series, n.5, 2011.

Disponível em: https://www.cream-migration.org/publ_uploads/CDP_05_11.pdf. Acessado em fevereiro de 2019.

FREGUGLIA, R. S. Efeitos da Migração sobre os Salários no Brasil. Tese (Doutorado em Teoria Econômica) - Faculdade de Economia, Administração e Contabilidade, Universidade de São Paulo. São Paulo, 2007. 
GAMA, L. C. D.; MACHADO, A. F. Migração e Rendimentos no Brasil: análise dos fatores associados no período intercensitário 2000-2010. Estudos Avançados, v. 28, n. 81, p. 155-174, 2014. DOI: https://doi.org/10.1590/S0103-40142014000200011

GOLD, S. J.; NAWYN, S. J. Routledge International Handbook of Migration Studies. New York: Routledge, 2019.

HADDAD, M. B.; PASTRE, R. O Centro-Oeste Brasileiro e suas Transformações Econômicas de 1970 a 2012. Revista Brasileira de Desenvolvimento Regional, v. 3, n. 2, p. 033-054, 2016. DOI: https://doi.org/10.7867/2317-5443.2015V3N2P033-054.

HARRIS, J. R.; TODARO, M. P. Migration, unemployment and development: a twosector analysis. The American Economic Review, vol.60, n.1, p. 126-142, 1970. DOI: https://doi.org/10.2307/1807860.

HECKMAN, J. J. Sample Selection bias as a Specification Error. Econometrica: Journal of the econometric society, vol.47, n.1, p. 153-161, 1979.

DOI: https://doi.org/10.2307/1912352.

JOHNSON, J. H.; SALT, J. (Ed.). Labour Migration: the internal geographical mobility of labour in the developed world. New York: Routledge, 2018.

LEE, E. S. A Theory of Migration. Demography, v. 3, n. 1, p. 47-57, 1966. DOI: https://doi.org/10.2307/2060063.

LIMA, R. Mercado de Trabalho: o capital humano e a teoria da segmentação. Pesquisa e Planejamento Econômico, vol.10, n.1, abril de 1980.

MASSEY, D. S. et al. Theories of international migration: A review and appraisal. Population and Development Review, v. 19, n. 3, p. 431-466, 1993. DOI: https://doi.org/10.2307/2938462.

MINCER, J. Family migration decisions. Journal of Political Economy, v. 86, n. 5, p. 749-773, 1978. DOI: https://doi.org/10.3386/w0199.

RAVENSTEIN, E. G. As Leis das Migrações. In: MOURA, H. A. (org.) Migração Interna, Textos Selecionados. Fortaleza: BNB/ENTENE, p. 25-88, 722p, 1980.

RIGOTTI, J. I. R.; CAMPOS, J.; HADAD, R. M. Migrações Internas no Brasil: (des) continuidades regionais à luz do Censo Demográfico 2010. Revista Geografias, p. 8-24, 2017. Disponível em: https://periodicos.ufmg.br/index.php/geografias/article/view/13444. Acessado em fevereiro de 2019.

RODRIGUES, K. C. T. T.; MOURA, F. K. F.; DE SOUZA, S. D. C. I.; MAIA, K. Diferença Salarial Segundo a Condição de Migração e Sexo na Bahia. Revista Economia e Desenvolvimento, v.14; n.2; 2015.

JÚNIOR, E.R.; FILHO, N.M.; FERREIRA, P.C. Migração e Seleção e Diferenças Regionais de Renda no Brasil. Pesquisa e Planejamento Econômico, vol. 32, n. 3, 2005. 
SANTOS, M. A. Migração: uma revisão sobre algumas das principais teorias. CEDEPLAR/FACE/UFMG, Texto para Discussão, v. 1, n. 398, 2010. Disponível em: https://www.cedeplar.ufmg.br/publicacoes/textos-paradiscussao/textos/2010/661-398-migracao-uma-revisao-sobre-algumas-das-principaisteorias. Acessado em julho de 2019.

NETO, R.S.; AZZONI, C.; Disparidades Regionais de Renda no Brasil: Qual o Papel das Amenidades? - In: Anais do IX Encontro Regional de Economia - ANPEC Nordeste, 2004.

FILHO, L. A.S. Migração: inserção socioeconômica, condição de atividade e diferenciais de rendimentos no Brasil. Tese (Doutorado em Desenvolvimento Econômico) Instituto de Economia, Universidade Estadual de Campinas, Campinas, 2017.

FILHO, L. A. S.; MAIA, A. G.; LIMA, Y. C.; SILVA, W. G. Dinâmica Migratória e Diferenciais de Rendimentos no Rio Grande do Norte-2000/2010. Revista de Economia Mackenzie, 16(1), 2019.

FILHO, L. A. S.; ALVES, D. F.; JUNIOR, J. J. S. Desenvolvimento Socioeconômico no Centro-Oeste: Uma análise a partir dos Censos Demográficos de 2000 e 2010. RDERevista de Desenvolvimento Econômico, v. 1, n. 42, 2019.

VARTATIAN, T. P. Heckman Selection Models. Bryn Mawr GSSW publication, 2015.

WHITE, M. J. (Ed.). International Handbook of Migration and Population

Distribution. New York: Springer, 2016. 